\title{
Prediction of esophageal varices and variceal hemorrhage in patients with acute upper gastrointestinal bleeding
}

\author{
Don C Rockey, ${ }^{1}$ Alan Elliott, ${ }^{2}$ Thomas Lyles ${ }^{3}$
}

\begin{abstract}
- Additional material is published online only. To view please visit the journal online (http://dx.doi.org/10. 1136/jim-2015-000047).

${ }^{1}$ Department of Internal Medicine, Medical University of South Carolina,

Charleston, South Carolina, USA

${ }^{2}$ Department of Statistical Science, Southern Methodist University, Dallas, Texas, USA

${ }^{3}$ Digestive Health Associates of Texas, Bedford, Texas, USA
\end{abstract}

Correspondence to Dr Don C Rockey, Department of Internal Medicine, Medical University of South Carolina,

96 Jonathan Lucas Street, Suite 803, MSC 623, Charleston 29425, SC, USA; rockey@musc.edu

Accepted 8 January 2016 Published Online First 12 February 2016

Copyright (C) 2016 American Federation for Medical Research

\section{(1) crossanat $^{-1}$}

To cite: Rockey DC,

Elliott A, Lyles T. J Investig Med 2016;64:745-751.

\section{ABSTRACT}

In patients with upper gastrointestinal bleeding (UGIB), identifying those with esophageal variceal hemorrhage prior to endoscopy would be clinically useful. This retrospective study of a large cohort of patients with UGIB used logistic regression analyses to evaluate the platelet count, aspartate aminotransferase (AST) to platelet ratio index (APRI), AST to alanine aminotransferase (ALT) ratio (AAR) and Lok index (all non-invasive blood markers) as predictors of variceal bleeding in (1) all patients with UGIB and (2) patients with cirrhosis and UGIB. 2233 patients admitted for UGIB were identified; 1034 patients had cirrhosis (46\%) and of these, 555 patients (54\%) had acute UGIB due to esophageal varices. In all patients with UGIB, the

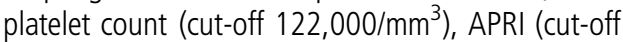
5.1), AAR (cut-off 2.8) and Lok index (cut-off 0.9) had area under the curve (AUC)s of 0.800 .82 , 0.64 , and 0.80 , respectively, for predicting the presence of varices prior to endoscopy. To predict varices as the culprit of bleeding, the platelet count (cut-off 69,000), APRI (cut-off 2.6), AAR (cut-off 2.5) and Lok Index (0.90) had AUCs of 0.76, 0.77, 0.57 and 0.73 , respectively. Finally, in patients with cirrhosis and UGIB, logistic regression was unable to identify optimal cut-off values useful for predicting varices as the culprit bleeding lesion for any of the non-invasive markers studied. For all patients with UGIB, non-invasive markers appear to differentiate patients with varices from those without varices and to identify those with a variceal culprit lesion. However, these markers could not distinguish between a variceal culprit and other lesions in patients with cirrhosis.

\section{INTRODUCTION}

The development of esophageal varices is a common clinical complication in patients with cirrhosis; severe bleeding from esophageal varices has been estimated to occur in approximately $30-40 \%$ of patients with cirrhosis ${ }^{1}$ and carries significant morbidity and mortality. ${ }^{2-6}$

Since the management of patients with cirrhosis, portal hypertension and esophageal variceal hemorrhage requires specific therapy, it is important to differentiate variceal bleeding from other causes of upper gastrointestinal bleeding (UGIB). First, patients with variceal hemorrhage require pharmacological therapy with an agent (ie, intravenous somatostatin

\section{Significance of this study}

\section{What is already known about this} subject?

- In patients with upper gastrointestinal bleeding (UGIB), identifying those with esophageal variceal hemorrhage prior to endoscopy would be clinically useful.

- Non-invasive markers of esophageal varices appear to be helpful in identifying large esophageal varices in stable (out)patients with known cirrhosis.

- Non-invasive markers of esophageal varices have not been examined in the setting of acute UGIB.

\section{What are the new findings?}

- The platelet count, AST to platelet ratio index, AST to ALT ratio and Lok index accurately predicted the presence of varices prior to endoscopy among the larger group of all patients with acute UGIB.

- These same non-invasive measures were slightly less accurate in predicting a variceal culprit lesion as the cause of bleeding.

- In patients with cirrhosis and UGIB, logistic regression was unable to identify optimal cut-off values useful for predicting varices as the culprit bleeding lesion for any of the non-invasive markers studied.

How might these results change the focus of research or clinical practice?

- The data suggest that in cirrhotics with UGIB, endoscopy is required to make a definitive diagnosis of variceal bleeding.

analogues or terlipressin) to reduce portal pressure. ${ }^{7-9}$ Prophylactic antibiotics are also indicated in these patients. ${ }^{70}$ Finally, it has been suggested that patients with esophageal varices should undergo endoscopy sooner than those without varices. ${ }^{4}$ Thus, rapid identification of patients with variceal hemorrhage would greatly enhance the care of this patient population.

Prediction of the presence of varices prior to endoscopy has previously been studied in stable 
outpatients, primarily by using non-invasive markers of portal hypertension, such as the platelet count, AST-ALT ratio (AAR), AST-platelet ratio index (APRI), Lok index, Forn's index, functional assays, ${ }^{11}$ transient elastography ${ }^{12}$ and other novel imaging techniques. ${ }^{13}{ }^{14}$ These markers have had variable correlation with the presence of varices when used in outpatients. ${ }^{11-17}$ However, they have not been studied in patients presenting with acute UGIB. Importantly, many of these indices utilize laboratory values that are routinely obtained as part of initial assessment and triage in patients with UGIB, making them attractive for routine clinical use.

In this study, we hypothesized that non-invasive markers of portal hypertension may be useful in determining the presence of varices, and also of varices as etiology of bleeding, prior to endoscopy. The aims of this study were therefore to determine whether readily available and practical clinical markers might predict the presence of varices prior to endoscopy in several different clinical contexts. First, in the entire population of all patients with acute UGIB, we sought to determine whether non-invasive markers could accurately predict the presence of varices (ie, in cirrhotic patients) prior to endoscopy. Further, we analyzed this group to determine whether non-invasive markers could accurately predict varices as the culprit bleeding lesion. Finally, in the specific cohort of patients with cirrhosis and UGIB, we sought to determine whether non-invasive markers could identify varices as the culprit bleeding lesion prior to endoscopy.

\section{METHODS}

This cohort study focused on non-invasive blood markers of portal hypertension to predict presence of varices and also variceal bleeding as etiology of acute UGIB. The study included patients admitted to Parkland Memorial Hospital (Dallas, Texas, USA), a University of Texas Southwestern (UTSW) teaching hospital, from January 1, 2003 through July 1, 2012. Inclusion required documented UGIB. Patients were identified retrospectively using the institution's chronic liver disease database (2003-2006) and UTSW's Gastrointestinal Bleeding Healthcare Registry (2007-2012); patient data in the latter registry are collected prospectively, and all patients admitted with UGIB from this institution were included in this cohort. Patients with incomplete clinical data (laboratory, endoscopy, or 30-day outcome) were excluded from the current analysis.

We first subdivided the main cohort into patients with cirrhosis and those without cirrhosis. Cirrhosis was defined as follows: a history consistent with chronic liver disease, plus the presence of clinical features consistent with cirrhosis, including clinical findings of cirrhosis and portal hypertension (spider angiomata, gynecomastia, splenomegaly, thrombocytopenia) or a documented complication of chronic liver disease (ie, ascites, varices, hepatic encephalopathy), and/or imaging consistent with cirrhosis, and/or liver histology consistent with cirrhosis.

UGIB was defined as reported or witnessed melena, hematemesis, coffee ground emesis or hematochezia (with a documented upper gastrointestinal tract lesion) in the setting of at least a four point drop in hematocrit from baseline or lower than normal. It is standard practice at our institution to perform esophagogastroduodenoscopy (EGD) in patients with any form of UGIB as defined above unless contraindications to endoscopy exist. We have developed a standardized approach to management of UGIB including institution of proton pump inhibitor infusion in all patients and adjunctive use of octreotide infusion and antibiotics in patients with cirrhosis. Standard operating procedure for practitioners is to use multimodality therapy for all highrisk lesions (ie, those with stigmata of recent bleeding or those actively bleeding). However, specific management was left to the individual attending physician.

Patients with UGIB were identified, and data related to the hospital admission were abstracted and entered into the chronic liver disease database and a Gastrointestinal Bleeding Healthcare Registry. Data captured included the following: clinical features associated with gastrointestinal bleeding, patient demographics, mortality and cause of death, medical history, packed red blood cell transfusions, hospital course data (intensive care unit stay, intubation, use of vasoactive agents), laboratory data on admission, vital signs at admission and endoscopic data (types of procedures performed, endoscopic diagnosis, stigmata of recent or active hemorrhage, and endoscopic therapies). A bleeding lesion in any given case was labeled as the primary diagnosis and the culprit gastrointestinal bleeding lesion. In some patients, more than one diagnosis was present (eg, esophagitis or gastritis was identified in addition to a primary lesion, and considered a secondary lesion) but not deemed to be the cause of hemorrhage. Variceal bleeding is deemed to occur when varices with stigmata of bleeding are present $^{7} 18$ when stigmata of variceal bleeding are present, varices were deemed to be the culprit lesion. For varices, additional data were collected such as number and grade of varices, use of band ligation as hemostasis and number of bands placed. Determination of bleed etiology and necessary treatment was determined by the attending physician at the time of the patient's endoscopic examination. For the purposes of analyses, patients with esophageal, but not gastric varices were considered to have variceal hemorrhage.

Demographics, laboratory values and physical examination findings are the initial values on the patient's admission to the hospital. APRI, AAR and Lok index were calculated using admission laboratory data, and in accordance with previously published formulas. ${ }^{19-21}$ Child-Pugh scores and model for end stage liver disease (MELD) scores were calculated using admission laboratory values, physical examination and clinical presentation. Endoscopic data were gathered at the time of endoscopy, and hospital course data were reviewed and collected after the hospitalization.

Patients were excluded from this analysis if they had a form of gastrointestinal bleeding other than an upper gastrointestinal source, age under 18, did not have endoscopy during their hospitalization, or were pregnant. For patients who had multiple hospitalizations for bleeding during the study period, rebleeding after 42-days from the index bleed was considered a new bleed. A readmission within 42-days from the index bleed was considered a rebleed and data from that admission were not captured in the study database. The study was approved by the University of Texas Southwestern Institutional Review Board and met all criteria for good clinical practice. ${ }^{22}$ 
Descriptive statistics were used to present demographic and clinical characteristics of patients in the study cohort. The primary outcomes of this study were identification of the presence of esophageal varices prior to EGD, identification of esophageal varices as the culprit lesion in patients presenting with acute UGIB, and identification of esophageal varices as the culprit lesion in patients with cirrhosis. We specifically aimed to examine previously reported noninvasive tests used to assess portal hypertension and varices.

\section{Statistics}

Descriptive statistics were used to present demographic and clinical characteristics of patients in the study cohort. The primary outcomes of this study was identification of the presence of esophageal varices prior to EGD, identification of varices as bleed etiology in patients presenting with acute UGIB and identification of varices as primary etiology of acute UGIB in patients with cirrhosis. We specifically aimed to examine previously published non-invasive, serum markers of portal hypertension and their correlation with esophageal varices. Student's $t$ test or analysis of variance was used to compare means by group. $\chi^{2}$ Analysis was used to compare categorical data by group. To reduce the effect of outliers in the data set, 5\% Winsorization was applied to the data set as a whole in order to adjust extreme values and provide more robust estimation for statistical inference. ${ }^{23}$ Univariate logistic regression was used to determine which variables were predictive of the outcome variables with area under the curve (AUC) and measures of sensitivity and specificity used to assess the variable's predictive capabilities. Predictive cut-off values for variables were determined using the logistic model to indicate what values of each independent variable predicts the outcome with greater than 50\% probability. Multiple logistic regression was used to determine which combination of variables was predictive of the outcomes. A $p$ value less than 0.05 was considered statistically significant. No adjustment of the $\mathrm{p}$ values was made for multiple tests. Analyses were performed using SAS V.9.3 (SAS Institute, Cary, North Carolina, USA).

\section{RESULTS}

We identified a total of 2233 unique admissions of patients with acute UGIB (figure 1). After 5\% Winsorization, the cohort included 1034 patients with cirrhosis, and 991 patients without cirrhosis. Of the patients with cirrhosis, $815(79 \%)$ were found to have esophageal varices at the time of endoscopy. Of these patients, 260 (25\% of the entire cirrhosis cohort) were found to have small or grade 1 varices (our institutional practice grades varices on a scale from 1 to 4 , per the Paquet scale ${ }^{24}$ ) that were not the causative etiology of bleeding. Thus, of the group of 1034 patients with cirrhosis, 555 (54\%) patients were classified as having bled from esophageal varices. The remaining 479 $(46 \%)$ patients from this group bled from other etiologies (figure 1, online supplementary table S1).

We first sought to examine differences between patients with cirrhosis and those without cirrhosis. Patients with cirrhosis and acute UGIB were more likely to be male, Hispanic in ethnicity, and had a higher in-hospital mortality rate. They also had several notable laboratory abnormalities including lower platelets, higher international

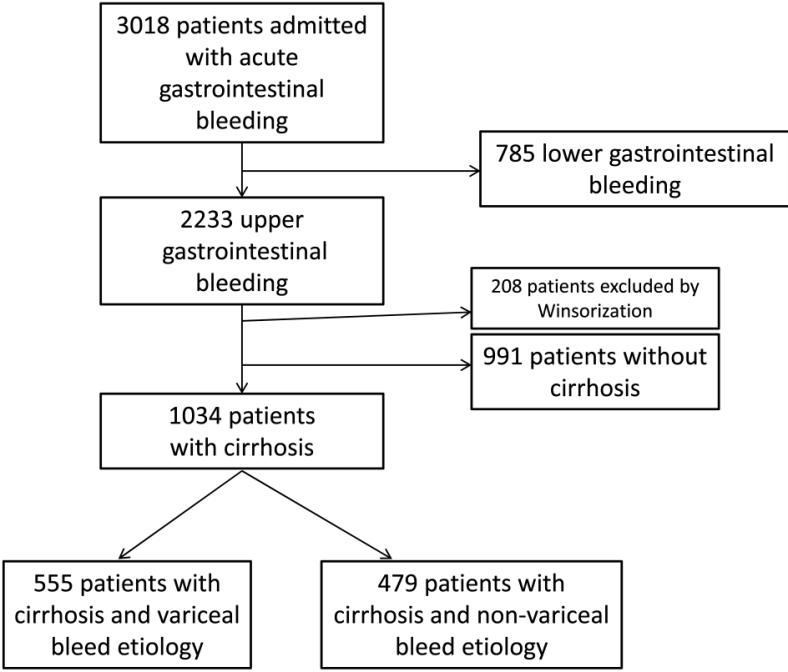

Figure 1 Flow diagram of the patient cohort. 3018 patients were admitted to our institution with acute gastrointestinal bleeding during the study period. After excluding patients with lower gastrointestinal bleeding and removing the upper and lower $5 \%$ (Winsorization), we divided the cohort into those with cirrhosis (1034 patients) and those without cirrhosis (991). We then further subdivided the cirrhosis cohort into variceal (555 patients) and non-variceal bleeding (479 patients) groups.

normalized ratio (INR), higher total bilirubin, higher AST, higher ALT (all $p<0.001$ for cirrhosis with or without variceal bleeding vs patients without cirrhosis) (table 1). As might be predicted, patients with cirrhosis had substantially different non-invasive markers of portal hypertension, such as APRI, Lok index and AAR than patients without cirrhosis (all $\mathrm{p}<0.001$ for cirrhosis with or without variceal bleeding vs patients without cirrhosis) (table 1). Notably, although both groups had similar hematocrit levels at times of admission, the mortality in patients with cirrhosis (both groups) had significantly higher mortality than patients without cirrhosis $(\mathrm{p}<0.001)$.

The cirrhosis cohort was then specifically examined to determine differences between cirrhosis patients with an esophageal variceal culprit lesion and those with a nonvariceal culprit lesion. The two groups had similar causes of cirrhosis and similar distribution across the Child-Turcotte-Pugh classification. However, there were several clinical and laboratory features that appeared to be different (table 2); patients with variceal bleeding had lower systolic blood pressure and lower mean arterial pressure. Interestingly, those with non-variceal bleeding had higher MELD and MELD-Na (sodium) scores (table 2). Several of the non-invasive markers had statistically significant differences between the variceal and non-variceal cirrhotics. Patients with variceal bleeding had lower platelet counts, a lower Lok index and a lower AAR. APRI was the same between the two groups.

\section{Prediction of the presence of esophageal varices in all patients with UGIB}

Logistic regression analysis was used to determine the predictive ability of non-invasive markers to identify the presence of esophageal varices in all patients presenting with 
Table 1 Demographic, laboratory and clinical characteristics of all patients presenting with UGIB

\begin{tabular}{|c|c|c|c|}
\hline & \multicolumn{2}{|c|}{$\begin{array}{l}\text { Patients with cirrhosis } \\
(n=1034)\end{array}$} & \multirow[b]{2}{*}{$\begin{array}{l}\text { Patients } \\
\text { without } \\
\text { cirrhosis } \\
(n=991)\end{array}$} \\
\hline & $\begin{array}{l}\text { Esophageal } \\
\text { variceal } \\
\text { bleeding } \\
(n=555)\end{array}$ & $\begin{array}{l}\text { Non-variceal } \\
\text { bleeding } \\
(\mathrm{N}=479)\end{array}$ & \\
\hline Age-years (SD) & $50(9)$ & $52(10)$ & $53(15)$ \\
\hline Gender-female (\%) & $125(23)$ & $163(34)$ & $351(35)$ \\
\hline \multicolumn{4}{|l|}{ Ethnicity } \\
\hline Hispanic & $305(55 \%)$ & $212(44 \%)$ & $329(33 \%)$ \\
\hline White & $164(30 \%)$ & $170(36 \%)$ & $261(27 \%)$ \\
\hline African-American & $67(12 \%)$ & $85(18 \%)$ & $369(37 \%)$ \\
\hline Other & $19(3 \%)$ & $12(2 \%)$ & $32(3 \%)$ \\
\hline In-hospital mortality & $40(7.0 \%)$ & $29(7.0 \%)$ & $66(7 \%)$ \\
\hline WBCs (×109/L) & $9( \pm 6)$ & $9( \pm 5)$ & $11( \pm 6)$ \\
\hline Hematocrit (\%) & $27( \pm 7)$ & $28( \pm 8)$ & $29( \pm 8)$ \\
\hline Platelets (×109/L) & $114( \pm 61)$ & $133( \pm 89)$ & $240( \pm 119)$ \\
\hline BUN (mg/dL) & $25( \pm 17)$ & $25( \pm 18)$ & $33( \pm 16)$ \\
\hline Sodium (mmol/L) & $135( \pm 5)$ & $135( \pm 5)$ & $136( \pm 5)$ \\
\hline Creatinine (mg/dL) & $1( \pm 0.7)$ & $1.2( \pm 1.2)$ & $2.1( \pm 9.0)$ \\
\hline INR & $1.5( \pm 0.5)$ & $1.5( \pm 0.9)$ & $1.3( \pm 0.9)$ \\
\hline Total bilirubin (mg/dL) & $2.7( \pm 4.4)$ & $2.8( \pm 4.4)$ & $0.7( \pm 1.3)$ \\
\hline Albumin (g/dL) & $2.9( \pm 0.6)$ & $2.9( \pm 0.7)$ & $\mathrm{n} / \mathrm{a}$ \\
\hline AST & $72( \pm 44)$ & $71( \pm 44)$ & $54( \pm 137)$ \\
\hline ALT & $45( \pm 33)$ & $39( \pm 32)$ & $32( \pm 35)$ \\
\hline APRI & $1.8( \pm 1.9)$ & $1.7( \pm 1.9)$ & $0.5( \pm 1.6)$ \\
\hline Lok Index & $0.89( \pm 0.14)$ & $0.84( \pm 0.25)$ & $0.5( \pm 0.3)$ \\
\hline AST:ALT ratio & $1.8( \pm 0.9)$ & $2.1( \pm 1.1)$ & $1.5( \pm 0.8)$ \\
\hline
\end{tabular}

ALT, alanine aminotransferase; AST, aspartate aminotransferase; APRI, AST to platelet ratio index; BUN, blood urea nitrogen; INR, international normalized ratio; n/a, not applicable; UGIB, upper gastrointestinal bleeding; WBC, white blood cell.

UGIB. By performing AUC analyses, we determined optimal cut-off values. For all patients presenting with UGIB, the platelet count (cut-off $122,000 / \mathrm{mm}^{3}$ ), APRI (cut-off 5.1), AAR (cut-off 2.81) and the Lok index (cut-off 0.86 ) had AUCs of $0.800 .83,0.64$, and 0.80 , respectively, for predicting the presence of esophageal varices prior to endoscopy. When we considered simple identification of the presence of esophageal varices, and associated AUC values, we created a summary of best fit performance characteristics (table 3). Of the four non-invasive approaches studied, AAR and APRI had poor specificity. Platelets and the Lok index had similar sensitivities, specificities, positive predictive value and negative predictive value.

Although we determined optimal cut-off values through best-fit analysis of the AUC, we explored other cut-off values for each non-invasive marker, with corresponding unique sensitivities and specificities (table 4).

\section{Prediction of esophageal varices as the culprit lesion in patients with UGIB}

We utilized logistic analysis to examine the predictive ability of non-invasive markers to predict esophageal varices as the bleeding etiology in patients presenting with UGIB (table 5). In this patient subset, the platelet count
Table 2 Characteristics of patients with cirrhosis presenting with UGIB

\begin{tabular}{|c|c|c|c|}
\hline & $\begin{array}{l}\text { Esophageal } \\
\text { variceal } \\
\text { etiology }(n=555)\end{array}$ & $\begin{array}{l}\text { Non-variceal } \\
\text { etiology } \\
(n=479)\end{array}$ & $p$ value \\
\hline Systolic blood pressure & $120( \pm 23)$ & $124( \pm 22)$ & 0.006 \\
\hline Mean arterial pressure & $86( \pm 16)$ & $89( \pm 16)$ & 0.011 \\
\hline Pulse & $97( \pm 20)$ & $94( \pm 21)$ & 0.144 \\
\hline $\begin{array}{l}\text { Ascites present on } \\
\text { physical examination }\end{array}$ & $137(25 \%)$ & $86(18 \%)$ & 0.01 \\
\hline Cirrhosis etiology & & & 0.63 \\
\hline Alcohol & $195(35 \%)$ & $162(39 \%)$ & \\
\hline Hepatitis C & $270(49 \%)$ & $187(45 \%)$ & \\
\hline Hepatitis B & $31(6 \%)$ & $21(5 \%)$ & \\
\hline NAFLD & $22(4 \%)$ & $21(5 \%)$ & \\
\hline Cryptogenic & $22(4 \%)$ & $16(4 \%)$ & \\
\hline Other & $15(3 \%)$ & $7(2 \%)$ & \\
\hline CTP class & & & 0.41 \\
\hline A & $187(34 \%)$ & $124(30 \%)$ & \\
\hline B & $257(46 \%)$ & $197(48 \%)$ & \\
\hline $\mathrm{C}$ & $111(20 \%)$ & $93(22 \%)$ & \\
\hline MELD & $14( \pm 6)$ & $15( \pm 7)$ & 0.002 \\
\hline MELD-Na & $17( \pm 6)$ & $18( \pm 7)$ & 0.007 \\
\hline Platelets & $114( \pm 61)$ & $133( \pm 89)$ & $<0.001$ \\
\hline APRI & $1.8( \pm 1.9)$ & $1.7( \pm 1.9)$ & 0.67 \\
\hline Lok index & $0.89( \pm 0.14)$ & $0.84( \pm 0.25)$ & $<0.001$ \\
\hline AAR & $1.8( \pm 0.9)$ & $2.1( \pm 1.1)$ & $<0.001$ \\
\hline
\end{tabular}

(cut-off 69,000), APRI (cut-off 2.6), AAR (cut-off 2.5) and Lok Index (0.90) had AUCs of 0.76, 0.77, 0.57 and 0.73 , respectively. For prediction of bleeding from varices, specificity was marginal for all markers, with the Lok index having the best specificity at $63 \%$. Overall, the platelet count and Lok index had the best test performances of the four markers. A cut-off platelet count for the presence of varices was determined to be less than or equal to $69,000 /$ $\mathrm{mm}^{3}$, while a cut-off Lok index for the prediction of varices was determined to be less than or equal to 0.9 .

Table 3 Best fit performance of non-invasive markers for prediction of the presence of esophageal varices in all patients with UGIB

\begin{tabular}{lclll}
\hline & Platelets & AST:ALT ratio & APRI & Lok index \\
\hline AUC (95\% CI) & 0.80 & 0.64 & 0.83 & 0.80 \\
Cut-off & 122 & 2.81 & 5.1 & 0.86 \\
Sensitivity (\%) & 80 & 93 & 96 & 73 \\
Specificity (\%) & 63 & 15 & 13 & 77 \\
PPV (\%) & 78 & 64 & 64 & 84 \\
NPV (\%) & 61 & 57 & 64 & 64 \\
Accuracy (\%) & 74 & 64 & 64 & 75 \\
LR+ & 2.1 & 1.1 & 1.1 & 3.2 \\
LR- & 0.4 & 0.5 & 0.3 & 0.4 \\
\hline
\end{tabular}

AUC, area under the curve; $L R+$, Positive likelihood ratio; $L R-$, negative likelihood ratio; NPV, negative predictive value; PPV, positive predictive value; UGIB, upper gastrointestinal bleeding. 
Table 4 Cut-off values of non-invasive markers for prediction of esophageal varices prior to endoscopy in all patients with UGIB

\begin{tabular}{lclc}
\hline Non-invasive marker & Cut-off & Sensitivity (\%) & Specificity (\%) \\
\hline Platelets & 58 & 95 & 17 \\
& 80 & 87 & 43 \\
& 142 & 71 & 77 \\
AST-ALT ratio & 176 & 61 & 88 \\
& 3.7 & 97 & 6 \\
& 1.9 & 77 & 40 \\
APRI & 1.3 & 55 & 64 \\
& 0.9 & 22 & 92 \\
& 11.4 & 99 & 2 \\
& 7.9 & 98 & 6 \\
Lok index & 3.8 & 88 & 37 \\
& 1.8 & 73 & 76 \\
& 0.93 & 79 & 63 \\
& 0.78 & 68 & 85 \\
& 0.70 & 63 & 90 \\
\hline
\end{tabular}

APRI, AST to platelet ratio index; UGIB, upper gastrointestinal bleeding.

\section{Prediction of esophageal varices as the culprit lesion in cirrhotic patients with UGIB}

We next attempted to identify ideal cut-off values for detection of bleeding esophageal varices in cirrhotic patients presenting with acute UGIB. Although there was a statistically significant difference in the measured noninvasive markers between patients with cirrhosis with variceal bleeding, and patients with cirrhosis and non-variceal bleeding, logistic regression was unable to clinically differentiate variceal from non-variceal bleeding. Overall, the examined markers had poor AUCs, poor sensitivities and clinically irrelevant cut-offs (table 6).

\section{DISCUSSION}

Acute UGIB is a common clinical problem in the USA, with an annual incidence of $82-146$ cases per 100,000 adults ${ }^{25}$ and estimated medical costs of $\$ 1$ billion. ${ }^{26}$ Accordingly, accurate identification of UGIB and appropriate triage of care is essential in the management of these patients. A variety of clinical features assist the clinician in triage,

Table 5 Performance of non-invasive markers for prediction of esophageal varices as the bleeding etiology in all patients with UGIB

\begin{tabular}{lllll}
\hline & Platelets & AST:ALT ratio & APRI & Lok index \\
\hline AUC & 0.76 & 0.57 & 0.77 & 0.73 \\
Cut-off & 69 & 2.5 & 2.6 & 0.9 \\
Sensitivity & 91 & 85 & 92 & 70 \\
Specificity (\%) & 24 & 19 & 21 & 63 \\
PPV (\%) & 76 & 73 & 76 & 83 \\
NPV (\%) & 49 & 31 & 49 & 44 \\
Accuracy (\%) & 72 & 67 & 72 & 68 \\
LR+ & 1.2 & 1.0 & 1.2 & 1.9 \\
LR- & 0.4 & 0.8 & 0.4 & 0.5 \\
\hline
\end{tabular}

APRI, AST to platelet ratio index; AUC, area under the curve; NPV, negative predictive value; PPV, positive predictive value; $L R+$, Positive likelihood ratio; LR-, negative likelihood ratio; UGIB, upper gastrointestinal bleeding.
Table 6 Performance of non-invasive markers for prediction of esophageal varices as the bleeding etiology in cirrhotics with UGIB

\begin{tabular}{lllll}
\hline & Platelets & AST:ALT ratio & APRI & Lok index \\
\hline AUC & 0.50 & 0.62 & 0.52 & 0.56 \\
Cut-off & 461 & 2.8 & 10.5 & 1 \\
Sensitivity & $0.2 \%$ & $22 \%$ & $0.2 \%$ & $0 \%$ \\
Specificity (\%) & 100 & 88 & 99 & 100 \\
\hline \multicolumn{2}{l}{ AUC, area under the curve; UGIB, upper gastrointestinal bleeding. }
\end{tabular}

including history, examination, and laboratory data. While medical history may be known for many patients at the time of admission, a subset of patients present without a known medical history. For example, some patients with acute UGIB are not known to have cirrhosis at the time of presentation; in a previous study, the diagnosis of cirrhosis was unknown in $35 \%$ of patients at the time of presentation with acute UGIB. ${ }^{27}$ This is especially important as in-hospital mortality for cirrhotics with acute UGIB is greater than for patients without cirrhosis, ${ }^{28}$ and it is possible that patients with cirrhosis may benefit from early endoscopy. $^{26}$

We found that in patients with known cirrhosis, esophageal variceal hemorrhage caused bleeding in approximately half of patients. Thus, the precise etiology of bleeding is often unknown until the time of endoscopy. In this study, we have shown that in a large combined cohort of patients presenting to an emergency room with acute UGIB, the platelet count was the best predictor of esophageal varices being present on endoscopy. In comparison to platelets, the Lok Index had a similar negative predictive value (64\%) and a higher positive predictive value $(84 \%)$ for the prediction of the presence of esophageal varices in patients presenting with acute UGIB.

In patients with cirrhosis, however, non-invasive markers of portal hypertension were unable to differentiate esophageal variceal from non-variceal bleeding prior to endoscopy. On examination of the cirrhosis cohort (table 2), there was a significant difference between the platelet count, APRI and Lok. However, further analysis with logistic regression deemed these markers unable to accurately identify varices as bleeding etiology prior to endoscopy.

While non-invasive markers of portal hypertension have previously been studied in the context of esophageal variceal screening in high-risk patients ${ }^{12} 29$ and correlation with fibrosis, they have not been applied to patients presenting with acute UGIB. Further, with the exception of platelet count, the respective non-invasive marker indices examined in this study were formulated using chronic hepatitis $\mathrm{C}$ virus patient cohorts. Thus, this is a novel use of previously reported indices.

Although the Lok Index is calculated with AST, ALT, platelets, and INR (all of which can be easily obtained and reported in a time efficient manner), it requires a dedicated calculation; whereas the platelet count is readily obtainable and easily interpretable. Thus, we speculate that the application of the platelet count in the initial assessment and triage of patients with acute UGIB could potentially augment a clinician's initial decision-making by rapidly 
identifying those patients likely to have portal hypertension, and thus in need of adjunctive octreotide, antibiotics and potential transfer to facilities that have gastroenterology services on call. Unfortunately, while reasonably sensitive, this clinical measure is not specific.

There are several strengths of this study, notably the size of the overall patient population, and the size of the cirrhosis cohort. Furthermore, data were collected over the course of almost a decade, at a single center, during a time in which standard of care did not substantially change, (ie, the use of proton pump inhibitors, vasoactive agents (octreotide) and antibiotics in cirrhosis presenting with UGIB). Further, data collection was complete since the premise of the study is to utilize routine, easily obtainable laboratory markers that are commonly drawn at the time of an initial assessment.

We recognize limitations of this study. The study was at a single center, was retrospective, and not all patients diagnosed with cirrhosis had a biopsy proven diagnosis. However, practice at our center followed closely currently published guidelines suggesting that the practices followed are generalizable nationally. ${ }^{70}{ }^{31}$ Additionally, our population was extremely heterogeneous, suggesting that the findings are likely generalizable to a wide range of patients. With regard to concern about a biopsy diagnosis of cirrhosis, it should be emphasized that we used commonly accepted clinical criteria for the diagnosis of cirrhosis. Interestingly, mortality in our cohort was comparatively low in comparison with other studies, ${ }^{32}{ }^{33}$ raising concerns about differences in care provided at our institution compared to other institutions. However, this should have no bearing on the conclusions drawn here since we focused on diagnosis rather than outcome.

In conclusion, by examining an extremely large, diverse and carefully phenotyped cohort of patients with UGIB, we have shown that patients with cirrhosis frequently bleed from lesions other than esophageal varices. We have also demonstrated that patients with cirrhosis have a higher mortality rate than those without cirrhosis and that the mortality rate in patients with cirrhosis with esophageal variceal and non-variceal bleeding was equivalent. This underscores that other factors, such as underlying severity of liver disease are clinically important, as is the clinical course of a patient with cirrhosis, especially if they develop other poor prognostic signs such as sepsis or multiorgan failure. Finally, while our study indicates that the platelet count may be potentially useful in augmenting a clinician's ability to quickly identify patients with cirrhosis prior to endoscopy, we conclude that non-invasive tests are not able to accurately predict whether the cause of bleeding is due to esophageal varices. In this setting, endoscopy is required to make a definitive diagnosis.

Acknowledgements The authors thank Rebecca Chason for her many contributions to this research, including maintenance of the Gastrointestinal Bleeding Healthcare Registry and data base. The author thank the housestaff, fellows, faculty, nurses, and staff of the Parkland Endoscopy unit for their diligence in care for patients with gastrointestinal bleeding.

Contributors DCR was involved in the study concept and design; acquisition of data; analysis and interpretation of data; drafting of the manuscript; critical revision of the manuscript for important intellectual content; statistical analysis. AE was involved in the analysis and interpretation of data; drafting of the manuscript; critical revision of the manuscript for important intellectual content; statistical analysis. TL was involved in the study concept and design; acquisition of data; analysis and interpretation of data; drafting of the manuscript; critical revision of the manuscript for important intellectual content.

Competing interests None declared.

Ethics approval UTSW IRB.

Provenance and peer review Not commissioned; externally peer reviewed.

\section{REFERENCES}

1 Jensen DM. Endoscopic screening for varices in cirrhosis: findings, implications, and outcomes. Gastroenterology 2002;122:1620-30.

2 Lyles T, Elliott A, Rockey DC. A risk scoring system to predict in-hospital mortality in patients with cirrhosis presenting with upper gastrointestinal bleeding. J Clin Gastroenterol 2014;48:712-20.

3 Cheung J, Soo I, Bastiampillai $R$, et al. Urgent vs. non-urgent endoscopy in stable acute variceal bleeding. Am J Gastroenterol 2009;104:1125-9.

4 Hsu YC, Chung CS, Tseng CH, et al. Delayed endoscopy as a risk factor for in-hospital mortality in cirrhotic patients with acute variceal hemorrhage. J Gastroenterol Hepatol 2009;24:1294-9.

5 Ananthakrishnan AN, McGinley EL, Saeian K. Outcomes of weekend admissions for upper gastrointestinal hemorrhage: a nationwide analysis. Clin Gastroenterol Hepatol 2009;7:296-302e1.

6 Myers RP, Papay KD, Shaheen AA, et al. Relationship between hospital volume and outcomes of esophageal variceal bleeding in the United States. Clin Gastroenterol Hepatol 2008;6:789-98.

7 Garcia-Tsao G, Sanyal AJ, Grace ND, et al. Prevention and management of gastroesophageal varices and variceal hemorrhage in cirrhosis. Hepatology 2007;46:922-38.

8 Azam Z, Hamid S, Jafri W, et al. Short course adjuvant terlipressin in acute variceal bleeding: a randomized double blind dummy controlled trial. J Hepatol 2012;56:819-24.

9 Ioannou GN, Doust J, Rockey DC. Systematic review: terlipressin in acute oesophageal variceal haemorrhage. Aliment Pharmacol Ther 2003;17:53-64

10 Mayorga CA, Rockey DC. Clinical utility of a standardized electronic order set for the management of acute upper gastrointestinal hemorrhage in patients with cirrhosis. Clin Gastroenterol Hepatol 2013;11:1342-8.

11 Lisotti A, Azzaroli F, Buonfiglioli F, et al. Indocyanine green retention test as a noninvasive marker of portal hypertension and esophageal varices in compensated liver cirrhosis. Hepatology 2014;59:643-50.

12 Sebastiani G, Tempesta D, Fattovich G, et al. Prediction of oesophageal varices in hepatic cirrhosis by simple serum non-invasive markers: results of a multicenter, large-scale study. J Hepatol 2010;53:630-8.

13 Takuma Y, Nouso K, Morimoto Y, et al. Measurement of spleen stiffness by acoustic radiation force impulse imaging identifies cirrhotic patients with esophageal varices. Gastroenterology 2013;144:92-101.e2.

14 Park Y, Kim SU, Park SY, et al. A novel model to predict esophageal varices in patients with compensated cirrhosis using acoustic radiation force impulse elastography. PLOS ONE 2015;10:e0121009.

15 Colecchia A, Montrone L, Scaioli E, et al. Measurement of spleen stiffness to evaluate portal hypertension and the presence of esophageal varices in patients with HCV-related cirrhosis. Gastroenterology 2012;143:646-54.

16 Shi KQ, Fan YC, Pan ZZ, et al. Transient elastography: a meta-analysis of diagnostic accuracy in evaluation of portal hypertension in chronic liver disease. Liver Int 2013;33:62-71.

17 Berzigotti A, Seijo S, Arena U, et al. Elastography, spleen size, and platelet count identify portal hypertension in patients with compensated cirrhosis Gastroenterology 2013;144:102-111.e1.

18 Sharara Al, Rockey DC. Gastroesophageal variceal hemorrhage. N Engl J Med 2001;345:669-81.

19 Wai CT, Greenson JK, Fontana RJ, et al. A simple noninvasive index can predict both significant fibrosis and cirrhosis in patients with chronic hepatitis C. Hepatology 2003;38:518-26.

20 Giannini E, Risso D, Botta F, et al. Validity and clinical utility of the aspartate aminotransferase-alanine aminotransferase ratio in assessing disease severity and prognosis in patients with hepatitis $C$ virus-related chronic liver disease. Arch Intern Med 2003;163:218-24.

21 Lok AS, Ghany MG, Goodman ZD, et al. Predicting cirrhosis in patients with hepatitis $C$ based on standard laboratory tests: results of the HALT-C cohort. Hepatology 2005;42:282-92.

22 [No authors listed]. World Medical Association Declaration of Helsinki: recommendations guiding physicians in biomedical research involving human subjects. JAMA 1997;277:925-6. 
23 Kotz S, Balakrishnan N, Read CB, et al. Encyclopedia of statistal sciences. Hoboken, NJ: Wiley-Interscience, 2006.

24 Paquet KJ. Prophylactic endoscopic sclerosing treatment of the esophageal wall in varices - a prospective controlled randomized trial. Endoscopy 1982;14:4-5.

25 Zhao Y, Encinosa W. Hospitalizations for gastrointestinal bleeding in 1998 and 2006: statistical brief \#65. Healthcare cost and Utilization Project (HCUP) statistical Briefs. Rockville, MD, 2006.

26 Wysocki JD, Srivastav S, Winstead NS. A nationwide analysis of risk factors for mortality and time to endoscopy in upper gastrointestinal haemorrhage. Aliment Pharmacol Ther 2012;36:30-6.

27 del Olmo JA, Peña A, Serra MA, et al. Predictors of morbidity and mortality after the first episode of upper gastrointestinal bleeding in liver cirrhosis. J Hepatol 2000;32:19-24.

28 Lecleire S, Di Fiore F, Merle V, et al. Acute upper gastrointestinal bleeding in patients with liver cirrhosis and in noncirrhotic patients: epidemiology and predictive factors of mortality in a prospective multicenter population-based study. J Clin Gastroenterol 2005;39:321-7.
29 Castéra L, Le Bail B, Roudot-Thoraval F, et al. Early detection in routine clinical practice of cirrhosis and oesophageal varices in chronic hepatitis C: comparison of transient elastography (FibroScan) with standard laboratory tests and non-invasive scores. J Hepatol 2009;50: $59-68$.

30 de Franchis R. Updating consensus in portal hypertension: report of the Baveno III Consensus Workshop on definitions, methodology and therapeutic strategies in portal hypertension. J Hepatol 2000;33:846-52.

31 de Franchis R. Evolving consensus in portal hypertension. Report of the Baveno IV consensus workshop on methodology of diagnosis and therapy in portal hypertension. J Hepatol 2005;43:167-76.

32 D'Amico G, De Franchis R., Cooperative Study Group. Upper digestive bleeding in cirrhosis. Post-therapeutic outcome and prognostic indicators. Hepatology 2003;38:599-612.

33 González-González JA, García-Compean D, Vázquez-Elizondo G, et al. Nonvariceal upper gastrointestinal bleeding in patients with liver cirrhosis. Clinical features, outcomes and predictors of in-hospital mortality. A prospective study. Ann Hepatol 2011;10:287-95. 